\title{
GABARAP suppresses EMT and breast cancer progression via the AKT/mTOR signaling pathway
}

Ying Liu

Tumor Hospital of Harbin Medical University

\section{Dandan Wang}

Tumor Hospital of Harbin Medical University

Mengxia Lei

Tumor Hospital of Harbin Medical University

Jiayi Gao

Tumor Hospital of Harbin Medical University

\section{Yuqing Cui}

Tumor Hospital of Harbin Medical University

Xiaoying Jin

Tumor Hospital of Harbin Medical University

\section{Qiujie Yu}

Tumor Hospital of Harbin Medical University

\section{Ying Jiang}

Mudanjiang Medical University

\section{Yan Guo}

Tumor Hospital of Harbin Medical University

Yali Liu

Tumor Hospital of Harbin Medical University

\section{Xuesong Chen}

Tumor Hospital of Harbin Medical University

\section{Li Cai ( $\nabla$ caili@ems.hrbmu.edu.cn )}

Tumor Hospital of Harbin Medical University

https://orcid.org/0000-0001-9666-8926

\section{Research}

Keywords: GABARAP, breast cancer, EMT, prognosis, AKT/mTOR pathway

Posted Date: May 5th, 2020

DOI: https://doi.org/10.21203/rs.3.rs-26093/v1 
License: (c) (i) This work is licensed under a Creative Commons Attribution 4.0 International License. Read Full License

Version of Record: A version of this preprint was published at Aging on February 11th, 2021. See the published version at https://doi.org/10.18632/aging.202510. 


\section{Abstract \\ Background}

Y-aminobutyric acid type $A$ (GABAA) receptor-associated protein (GABARAP) is rarely studied in tumor progression. Here, the authors investigated the expression and significance of GABARAP in breast cancer.

\section{Method:}

A large group of clinical samples was assessed to detect GABARAP expression and its associations with clinicopathological features and prognosis. Gain- and loss-of-function experiments in cell lines and mouse xenograft models were performed to elucidate the function and underlying mechanisms of GABARAP-regulated tumor progression.

\section{Results}

We analyzed GABARAP levels in clinical breast cancer samples and cell lines and confirmed that GABARAP was negatively correlated with advanced clinicopathologic features, such as tumor size $(\mathrm{P}=$ $0.025)$ and TNM stage $(P=0.001)$. Importantly, patients with low GABARAP levels had a poor prognosis $(p=0.0047)$. Functionally, our data revealed that GABARAP can inhibit proliferation, migration and invasion in vitro and in vivo. Importantly, low levels of GABARAP induced epithelial-mesenchymal transition (EMT), one of the most important mechanisms for the promotion of tumor metastasis, in breast cancer cells. Mechanistically, low levels of GABARAP increased the levels of p-AKT (S473) and pmTOR (S2448), and a specific AKT pathway inhibitor reversed the downregulation of GABARAP-induced tumor progression. In clinical breast cancer specimens, immunohistochemistry $(\mathrm{IHC})$ revealed that the distribution and intensity of GABARAP expression were negatively correlated with those of matrix metalloproteinase $(M M P) 2(P=0.0013)$ and MMP14 $(P=0.019)$.

\section{Conclusions}

Collectively, these data indicated that GABARAP suppressed the malignant behaviors of breast cancer cells, illuminating that the possible mechanism acts via the AkT/mTOR pathway. Targeting GABARAP may provide a potential diagnosis and treatment strategy for breast cancer.

\section{Background}

Breast cancer is the most common cancer in women and is the second leading cause of cancer-related death among females worldwide [1]. In China, the number of new breast cancer cases has continually risen in recent years, and the age of onset tends to be younger [2]. For patients with aggressive tumors that are refractory to multiple treatments, a dismal prognosis is expected, and a vast majority of these 
patients die of complications relating to metastatic diseases [3, 4]. Metastasis and chemoresistance lead to treatment failure, and the mechanisms responsible are poorly understood. Thus, it is essential to further characterize the molecular mechanism of breast cancer progression and to develop novel mechanism-based therapeutic strategies to block this process $[5,6]$. The purpose of this study was to evaluate the potential value of $\gamma$-aminobutyric acid type $A$ (GABAA) receptor-associated protein (GABARAP) as a therapeutic target for breast cancer metastasis.

GABARAP, located at $17 \mathrm{p} 13.1$, is a $14 \mathrm{kDa}$ cytoplasmic protein that plays an important role in the regulation of GABAA-receptor activity. It is a key player in the intracellular trafficking of these receptors [7, 8]. GABARBP binds to various intracellular proteins, which are all associated with vesicle transport processes, autophagy, apoptosis, which include the cytoskeleton, tubulin, gephyrin, the clathrin heavy chain, p130/phospholipase C-related inactive protein (PRIP), transferrin receptor, Unc-51-like kinase, Rasrelated protein 24, angiotensin II type 1 (AT1) receptor [9-14]. Recently, GABARAP has been reported to play a key role in inhibiting inflammation progression [15] and regulating angiogenic activity [16]. However, the expression and underlying mechanism of GABARAP in breast cancer are poorly understood. These observations have prompted us to speculate the role of GABARAP in the initiation and development of breast cancer.

The PI3K/AKT signaling pathway plays an important role in many cellular functions, including proliferation, adhesion, migration, invasion, metabolism, and survival. AKT has been reported to be overactivated in $>60 \%$ of cancer types [17-19], is involved in tumor development and is a potential target for tumor therapy. Epithelial-mesenchymal transition (EMT) is known to be a central mechanism responsible for the invasiveness and metastasis of various cancers $[20,21]$. The mechanism of EMT is complex and involves different signals from the tumor microenvironment, tumor cells and the interactions between these two components [22-24]. In this study, we explored the expression and biological functions of GABARAP using a comprehensive investigative approach that included clinical breast cancer samples along with cellular and animal models. Furthermore, we elucidated the mechanisms by which GABARAP suppressed EMT breast cancer cells, which partly occurs through the Akt/mTOR signaling pathway.

\section{Materials And Methods}

\section{Tissue specimens and patients}

This study was approved by the Ethics Committee of Harbin Medical University. Samples from 87 cases of histopathologically confirmed invasive ductal breast cancer (IDC) and 48 cases of ductal carcinoma in situ (DCIS) as well as 24 samples of normal breast tissue were included in this study. The IDC patients were female, hospitalized at the Affiliated Tumor Hospital of Harbin Medical University from March 2010 to November 2010 and followed-up until March 2015. The median follow-up was 58.9 months (range 16.8 to 63.3 months). Formalin-fixed paraffin-embedded (FFPE) tissues and complete clinical records of 
the patients were collected. None of the patients had undergone preoperative chemotherapy or radiotherapy.

\section{Cell culture}

The human breast cancer cell lines MDA-MB-231, MCF7, MDA-MB-468, T47D, UACC-812, MDA-MB-453, SKBR-3, and HCC70 and nontransformed breast cell line MCF-10A were obtained from the Cancer Research Institute of Heilongjiang Province, China. MCF7, T47D, UACC-812 and HCC70 cells were cultured in DMEM (Gibco, Carlsbad, CA, USA), whereas MDA-MB-453 and SKBR-3 cells were cultured in RPMI 1640 (Gibco, Carlsbad, CA, USA). All media for the abovementioned cancer cell lines were supplemented with 10\% fetal bovine serum (FBS) (PAN) (Gibco, Grand Island, NY, USA) and 1\% penicillin-streptomycin (Gibco, Grand Island, NY, USA). MCF-10A cells were maintained in DMEM-F12 (Gibco, Carlsbad, CA, USA) medium supplemented with hydrocortisone $(0.5 \mu \mathrm{g} / \mathrm{ml})$, insulin $(10 \mu \mathrm{g} / \mathrm{ml})$, hEGF $(20 \mathrm{ng} / \mathrm{ml})$ and $10 \% \mathrm{FBS}$. All cells were cultured in a humidified incubator at $37^{\circ} \mathrm{C}$ with $5 \%$ CO2. MDA-MB-231 and MDA-MB-468 cells were cultured in RPMI 1640 (Gibco, Carlsbad, CA, USA) supplemented with 10\% FBS and 1\% penicillinstreptomycin at $37^{\circ} \mathrm{C}$ in a humidified incubator without $\mathrm{CO} 2$.

\section{Cell transfection}

UACC-812 and T47D cells were infected with lentiviruses expressing specific shRNA to knock down GABARAP (GABARAP-shRNA). Human GABARAP targeted RNAi (RNAi: GCCUACAG UGACGAAAGUGTT) sequences was obtained from GeneChem Co. Ltd. (Shanghai, China). As a control, scrambled versions of these sequences(NC: GGCUCUAGAAAAGCCUAUGCdTdT) were used. The sequences shown above were inserted into the GV248 vector plasmid. For overexpression of GABARAP in MDA-MB-453 cells, full-length human GABARAP cDNA was cloned into the pLVX-puro vector. Lentiviral particles were constructed and packaged by Shanghai GeneChem Co. Ltd. Briefly, the cells were infected with lentivirus to generate stable cell lines. After $24 \mathrm{~h}$, the cells were transferred to medium containing $4 \mu \mathrm{g} / \mathrm{ml}$ puromycin and were cultured for 3 days.

\section{Cell viability assay}

The proliferation of T47D, UACC-812 and MDA-MB-453 cells proliferation assay was assessed with a Cell Counting Kit-8 (CCK-8) (Beyotime Institute of Biotechnology, Shanghai, China) according to the manufacturer's instructions. Briefly, 1000-3000 cells per well (depending on the cell type) were plated in each well of a 96 -well plate and cultured overnight. CCK-8 reagent ( $10 \mu \mathrm{L}$ per well) was added at 24,48 or $72 \mathrm{~h}$ after seeding and incubated at $37^{\circ} \mathrm{C}$ for $2 \mathrm{~h}$, at which point the absorbance of optical density (OD) value at $450 \mathrm{~nm}$ was measured. Three parallel experiments were performed for each assay with five replicate wells per condition.

\section{Cell invasion assays}

For the invasion assays, cells in serum-free medium were seeded into the upper chamber of a transwell insert (8- $\mu \mathrm{m}$ pore size, Millipore) coated with Matrigel (30ul 1:8 dilution, Sigma-Aldrich, USA). Medium 
containing $10 \%$ FBS was added to the lower chamber. After the cells were incubated at $37^{\circ} \mathrm{C}$ for $48 \mathrm{~h}$, noninvading cells that remained in the top chambers were removed with a cotton swab, and the cells that had migrated to the underside of the membrane were fixed in 100\% methanol for 30 min, air-dried, stained with $0.5 \%$ crystal violet (Sigma, St. Louis, MO, USA), imaged, and counted under a light microscope.

\section{Colony formation assay}

Cells were seeded into a 6 -well plate and cultured for 14 days in medium containing $10 \%$ FBS. Colonies were fixed in methanol for $30 \mathrm{~min}$, and $500 \mu \mathrm{L}$ of $0.5 \%$ crystal violet was added to each well for $30 \mathrm{~min}$ for visualization and counting.

\section{Wound healing assay}

For wound healing assays, cells cultured to $95 \%$ confluence in a 6-well plate were scratched with a $10-\mu \mathrm{L}$ pipette tip. Then, images of the wound were taken at $0 \mathrm{~h}$ and $24 \mathrm{~h}$. Mobility was calculated as follows: (mean area at $0 \mathrm{~h}-$ mean area at $\times \mathrm{h}) /($ mean area at $0 \mathrm{~h}) \times 100 \%$.

\section{Immunohistochemistry (IHC)}

The detailed experimental immunohistochemical procedures were described previously [25]. Antibodies against GABARAP (Proteintech, 11010-1-AP, dilution 1:100), E-cadherin (Proteintech, 20874-1-AP, dilution 1:4000), p-mTOR (Ser2448; Affinity, AF3308, dilution 1:50), matrix metalloproteinase (MMP) 2 (Proteintech, 10373-2-AP, dilution 1:200) and MMP14 (Proteintech, 14552-1-AP, dilution 1:200)were used for IHC.

\section{Western blot}

Cells were lysed using RIPA lysis buffer containing protease inhibitors to obtain protein. A BCA Protein Assay Kit was used to determine the protein concentrations. Proteins were separated by $10 \%$ SDSpolyacrylamide gel electrophoresis and transferred to PVDF membranes. Subsequently, the membranes were blocked with $5 \%$ BSA blocking reagent for 1 hour at RT and incubated with primary antibodies overnight at $4^{\circ} \mathrm{C}$. The next day, the membranes were washed and incubated for 1 hour at RT with secondary antibodies. Finally, the proteins were analysed using the ECL Plus kit. The antibodies used included the following: GABARAP (Abcam, ab109364, dilution 1:1000), E-cadherin (Abcam, ab40772, dilution 1:1000), N-cadherin (Abcam, ab76011, dilution 1:1000), Vimentin (Proteintech, 10366-1-AP, dilution 1:1000), MMP2 (Abcam, ab110186, dilution 1:1000), MMP14 (Abcam, ab3644, dilution 1:1000), AKT (Abcam, ab179463, dilution 1:1000), p-AKT (Ser473; Bioworld Technology, BS4007, dilution 1:1000), mTOR (Abcam, ab2732, dilution 1:1000), p-mTOR (Ser2448; Cell Signaling Technology, 5536, dilution 1:1000), p70S6K (Proteintech, 14485-1-AP, dilution 1:1000), and p-P70S6K (Thr389; Cell Signaling Technology, 9234, dilution 1:1000), MEK1/2 (Cell Signaling Technology, 8727, dilution 1:1000), p-MEK1/2 (Ser217/221; Cell Signaling Technology, 9154, dilution 1:1000), ERK1/2 (Cell Signaling Technology, 4695, dilution 1:1000), p-ERK1/2 (Thr202/Tyr204: Cell Signaling Technology, 4370, dilution 1:1000), IKK- $\beta$ (Cell Signaling Technology, 8943, dilution 1:1000), p-IKK- $\beta$ (Cell Signaling Technology, 2078, dilution 1:1000), 
ІкBa (Cell Signaling Technology, 4812, dilution 1:1000), p-ІкBa (Cell Signaling Technology, 2859, dilution 1:1000). Equal protein loading was assessed using a mouse anti- $\beta$-actin antibody (ZSGB-BIO, TA-09, dilution 1:1500).

\section{Nude mouse tumor xenograft model}

BALB/c athymic nude mice (female, 4-5 weeks old) were purchased from Beijing Vital River Laboratory Animal Technology Co., Ltd. and bred in pathogen-free conditions at the Animal Center of the Affiliated Tumor Hospital of Harbin Medical University. UACC-812/vector control or UACC-812/GABARAP-shRNA cells $\left(5 \times 10^{6}\right.$ cells in $100 \mu \mathrm{L}$ of PBS) were subcutaneously injected into the left flanks of mice in each group ( $n=10$ per group). When palpable tumors formed, the mice bearing UACC-812 cells with or without stable knockdown of GABARAP were randomized into two subgroups ( $\mathrm{n}=5$ per subgroup) and injected with LY294002 $(75 \mathrm{mg} / \mathrm{kg})$ or sterile water into the peritoneal cavity twice weekly for 3 weeks.

Subsequently, the tumor volume was monitored with Vernier calipers every five days for 3 weeks and calculated using the equation $\left(L \times W^{2}\right) / 2$, where $L$ is the length and $W$ is the width. Tumor weight was measured after excision on the final day of the experiment. After all the mice were sacrificed, a portion of the tumor tissue was fixed in formalin and embedded in paraffin for immunohistochemical analysis. All animal experiments were performed in accordance with the Institutional Animal Care and Use Committee (IACUC) of Harbin Medical University in China and the NIH Guidelines for the Care and Use of Laboratory Animals.

\section{Statistical analysis}

Statistical analyses were performed with SPSS 22.0 and GraphPad Prism software. The data are expressed as the means \pm standard deviations (SDs). All experiments were performed at least three times. The differences between two groups were analyzed with Student's tests and the $\chi 2$ test. The survival analysis was performed using Kaplan-Meier analysis and log-rank tests. Data from The Cancer Genome Atlas Breast Invasive Carcinoma (TCGA-BRCA) cohort were downloaded from the UALCAN Database (http://ualcan.path.uab.edu/index.html) and Oncomine Database (https://www.oncomine. org/resource/login.html); these data sets were used to detect GABARAP mRNA expression and survival analysis in breast cancer. A two-tailed $p$ value $<0.05$ was considered significant.

\section{Results}

\section{GABARAP is downregulated in primary breast cancer specimens as well as in breast cancer cell lines}

To investigate the potential role of GABARAP in breast cancer, we first analyzed GABARAP mRNA expression via breast cancer RNA-seq data from the TCGA database. We found that GABARAP expression was significantly downregulated in breast cancers compared with normal tissues $(P<0.0001$, Fig. $1 a)$. GABARAP expression was lower in all subtypes of breast cancer tissues than in normal tissues (Fig. 1b) $(P<0.0001)$. Moreover, the results of the TCGA data analysis were validated in 87 IDC samples, 48 DCIS samples and 24 noncancerous tissue samples via IHC (Fig. 1e). Positive staining (brown) was detected in 
the majority of noncancerous tissues (16/24) and in some DCIS tissues (17/48) but was detected less frequently in IDC tissues (27/87) ( $P<0.01$, Fig. 1f, Additional file 1: Table $S 1)$. This suggests that the low expression of GABARAP is related to the occurrence and development of breast cancer.

To test whether GABARAP was also decreased in cultured breast cancer cell lines, we conducted western blot analysis on eight breast cancer cell lines and nontransformed MCF-10A cells. As shown in Fig. $1 \mathrm{~g}$ and $h$, GABARAP displayed high expression levels in the nontransformed MCF-10A cells but only weak expression in T47D, UACC-812, and MCF-7 cells; furthermore, GABARAP had low expression in MDA-MB453, SKBR3, HCC70, MDA-MB-231 and MDA-MB-468 cells $(P<0.05)$. These results indicated that GABARAP is downregulated in breast cancer cells.

\section{The clinical significance of GABARAP in patients with breast cancer}

To further investigate whether low GABARAP expression is involved in breast cancer progression, we analyzed GABARAP mRNA expression based on RNA-seq data of individual cancer stages in breast cancer from the TCGA database (Fig. 1c). GABARAP downregulation was strongly associated with pTNM stage $(P<0.01)$. Then, we analyzed the correlation between GABARAP levels and the clinicopathological features of 87 patients with histopathologically confirmed IDC at Harbin Medical University Cancer Center (HMUCC). As shown in Additional file 1: Table S2, statistical analyses using the IHC results revealed that low levels of GABARAP in patients correlated with advanced $p$ T classification $(p=0.025)$, axillary lymph node metastasis $(P=0.023)$, advanced pTNM stage $(p=0.001)$, histological grade $(p=0.019)$ and ER status $(p=0.039)$. However, no significant association was found between GABARAP and age, Her-2 status, Ki-67 levels or P53 status. Therefore, we hypothesize that low expression of GABARAP is involved in tumor cell proliferation and plays an important role in breast cancer development. Additionally, survival analysis showed that patients with low levels of GABARAP expression had lower overall survival $(p=$ 0.0047) than those with high GABARAP expression levels (TCGA, Fig. 1d). These results demonstrate that GABARAP is expressed at low levels in breast cancer tissue and that low expression is associated with a poor prognosis for these patients.

\section{Low GABARAP levels enhance the malignant behavior of breast cancer cells}

We performed western blotting analysis to compare the expression levels of GABARAP in breast cancer cell lines and nontransformed MCF-10A cells. As shown in Fig. 1g, T47D and UACC-812 cells were used as GABARAP "loss-of-function" models, while MDA-MB-453 cells served as a GABARAP "gain-of-function" model. We detected the protein expression level of GABARAP in these target cells by western blot. As shown in Fig. 2a and b, compared with control cells, T47D and UACC-812 cells exhibited significant knockdown of GABARAP while MDA-MB-453 cells presented increased GABARAP expression (Fig. 3a).

To test whether GABARAP affects the proliferation of breast cancer cell lines, we conducted CCK8 assays and measured cell viability. Knockdown of GABARAP significantly accelerated cell growth in T47D and UACC-812 cells compared to the respective control cells (Fig. 2c and d), whereas overexpression of GABARAP in MDA-MB-453 cells attenuated cell growth (Fig. 3b). Accordingly, we observed similar 
patterns in the colony forming efficiency of GABARAP-overexpressing MDA-MB-453 cells (Fig. 3c) and GABARAP-shRNA-transfected T47D and UACC-812 cells (Fig. 2e and f), suggesting that GABARAP negatively regulates cell proliferation in vitro.

We further studied the potential effects of GABARAP on cell migration and invasion using wound healing and transwell assays, respectively. As shown in Fig. $2 \mathrm{~g}$ - j, both migration and invasion were significantly enhanced in T47D and UACC-812 cells with knockdown of GABARAP. However, overexpression of GABARAP in MDA-MB-453 cells markedly inhibited cell migration and invasion compared to MDA-MB-453 cells transfected with vector controls (Fig. $3 d$ and e) These results suggest that GABARAP inhibits cell migration and invasion in vitro.

\section{GABARAP regulates breast cancer progression via EMT}

EMT plays an important role in tumor metastasis, and we hypothesized that GABARAP affects the EMT process and inhibits breast cancer progression. Then, we analyzed the expression profile data of breast cancer patients clinical cohort from TCGA database. Pearson correlation was calculated among genes related to GABARAP, CDH1, MMP2, MMP14 and Akt/ mTOR signaling pathway. In result, the expression correlation of GABARAP and Akt/mTOR signaling pathway was identified in the clinical cohort of TCGA breast cancer patients, revealed the potential influence of GABARAP and Akt/mTOR signaling pathway. Furthermore, Akt/mTOR signaling pathway mediates genes related to epithelial mesenchymal transformation, such as CDH1, MMP2 and MMP14 (Fig. 4a). Here, we show that downregulation of GABARAP was associated with high levels of Vimentin, N-cadherin, MMP2 and MMP14 and low levels of E-cadherin (Fig. 4b). Conversely, GABARAP upregulation inhibited Vimentin, N-cadherin, MMP2 and MMP14 expression and upregulated E-cadherin expression (Fig. 4c). Taken together, these results indicate that GABARAP inhibits EMT and thereby suppresses breast cancer progression.

\section{Low levels of GABARAP induce EMT by activating the AKT/mTOR pathway}

Multiple pathways, including mTOR, PI3K/AKT and NF-KB signaling, are involved in GABARAP-regulated autophagy, inflammation and angiogenic activity [8-13]. To further verify how GABARAP regulates EMT, we measured AKT/mTOR, NF-KB and ERK/MAPK signaling in breast cancer cells. Knockdown of GABARAP in T47D and UACC-812 cells increased the levels of phosphorylated AKT, mTOR and p70s6k (Fig. $4 b$ ) but had no effect on p-ERK, p-MEK, p-IKK- $\beta$ and p-IKBa levels (Additional file 3: Fig. S1). By contrast, overexpression of GABARAP suppressed the phosphorylation of AKT, mTOR and p70s6k (Fig. 4c), and showed no effect on p-ERK, p-MEK, p-IKK- $\beta$ and p-IKBa levels (Additional file 3: Fig. S1). These results indicate that GABARAP levels are negatively correlated with the activation of AKT/mTOR pathways. Using pathway inhibitors, we found that GABARAP-mediated inhibition of invasion, migration, and EMT was reversed by the AKT pathway inhibitor LY-294002 (50 $\mu \mathrm{M}, 24$ h; Fig. 5a, b, c, d and e). Taken together, these data indicate that downregulation of GABARAP activates the AKT/mTOR pathway, which in turn promotes EMT of breast cancer cells. 
To evaluate the role GABARAP plays in regulating breast cancer progression in vivo, we established a xenograft tumor model in nude mice using UACC-812 cell lines stably transfected with vector control or GABARAP-shRNA and treated the animals with LY294002 $(75 \mathrm{mg} / \mathrm{kg})$ or sterile water. Mice were randomly assigned to the following experimental groups: vector control group, GABARAP-shRNA group, vector control + LY294002 group and GABARAP-shRNA + LY294002 group ( $\mathrm{n}=5$ per subgroup). (Fig. 6a). As expected, silencing GABARAP significantly increased tumor volumes and weight in mice compared with those in the control group. However, GABARAP-mediated inhibition of tumor formation was reversed by LY-294002 (Fig. 6b and c). Furthermore, tumors with GABARAP knockdown had a higher metastasis capacity, as evidenced by the increased MMP2 and p-mTOR staining and reduced E-cadherin staining (Fig. 6d). These results indicate that knocking down GABARAP suppresses breast cancer progression in vivo.

\section{GABARAP suppresses tumor progression in clinical breast cancer specimens}

To further examine the relationship between GABARAP and metastasis in human breast cancer, we performed IHC staining of GABARAP, MMP2 and MMP14 in 87 IDC specimens (Fig. 7a and c). Consistent with our observations in the tumor cell lines and xenograft models, the distribution and intensity of GABARAP were negatively correlated with MMP2 ( $P=0.0013$, Fig. 7b) and MMP14 ( $P=0.019$, Fig. $7 d)$ in breast cancer tissue specimens. Which clearly indicated that low GABARAP expression was associated with elevated metastasis in breast cancer patients.

\section{Discussion}

Metastasis and chemoresistance lead to treatment failure in breast cancer patients. Metastasis is a highly inefficient process, and the mechanisms involved are poorly understood [26, 27]. Thus, it is essential to further characterize the molecular mechanism(s) of breast cancer progression and to develop novel therapeutic strategies based on mechanisms to block breast cancer progression. Our study demonstrates that GABARAP is significantly downregulated in breast cancer tissues and that a low level of GABARAP is associated with a malignant breast cancer phenotype. Our findings indicate that, in terms of the mechanism, GABARAP inactivates the AKT/mTOR pathway and suppresses cell EMT. Clinically, we found that low levels of GABARAP were negatively associated with upregulation of the expression of MMPs, which we believe will serve as a promising prognostic indicator in breast cancer patients.

Recent studies have reported that GABARAP plays a crucial role in modulating the level of autophagy. For example, Sasai $\mathrm{M}$ et al. demonstrated that GABARAP, as an autophagy protein, plays an essential role in interferon-inducible GTPase-mediated host defense [28]. However, very little research on the role of GABARAP in tumor progression is available. Based on our assessment of a large number of clinical samples, the present study showed that GABARAP is significantly downregulated in breast cancer tissues, a result that is consistent with that of the public database. Moreover, we found that decreased GABARAP levels promoted cell proliferation, invasion and migration in vitro and promoted breast cancer growth and metastasis in vivo. Importantly, low levels of GABARAP induced EMT in breast cancer cells, which is one 
of the most important mechanisms for the progression of tumor metastasis. Some papers previously described the role of autophagy in the regulation of EMT. For example, Gugnoni M et al. previously demonstrated that CDH6 interacts with GABARAP promotes EMT and Thyroid tumor metastasis by restraining autophagy [29]. Akalay I et al. described that the role of autophagy in EMT in breast cancer [30]. While we will next study the role of GABARAP in EMT regulation is dependent of its role in autophagy or other.

A retrospective review of the literature indicates that GABARAP regulates multiple classical pathways, including the AKT/mTOR pathway. For example, Wu et al. showed that GABARAP promotes bone marrow mesenchymal stem cell-based osteoarthritis cartilage regeneration through the inhibition of the $\mathrm{PI} 3 \mathrm{~K} / \mathrm{AKT} / \mathrm{mTOR}$ signaling pathway [31]. Rho et al. reported that GABARBP inactivates cisplatin-induced dephosphorylation of the PI3K/Akt signaling pathway and promotes the sensitivity of ovarian cancer to apoptosis [14]. In line with these studies, we found that downregulation of GABARAP expression increased p-AKT and p-mTOR levels and induced EMT. Furthermore, EMT induced by low levels of GABARAP can be reversed by an AKT pathway inhibitor. We therefore conclude that low levels of GABARAP results in the activation of the AKT/mTOR signaling pathway.

\section{Conclusions}

Collectively, to the best of our knowledge, this is the first study to reveal a fundamental role of GABARAP in suppressing invasion, aggressiveness and metastasis, thus establishing GABARAP as a potential therapeutic target for the multifaceted progression of breast cancer.

\section{Abbreviations}

GABARAP

Y-aminobutyric acid type $A$ (GABAA) receptor-associated protein(GABARAP);

EMT

epithelial-mesenchymal transition(EMT);

IHC

immunohistochemistry (IHC);

MMP

matrix metalloproteinase (MMP);

PRIP

p130/phospholipase C-related inactive protein (PRIP)

AT1

angiotensin II type 1 (AT1);

IDC

invasive ductal breast cancer (IDC);

DCIS

ductal carcinoma in situ (DCIS);

Page $11 / 23$ 
FFPE

Formalin-fixed paraffın-embedded (FFPE);

TCGA

The Cancer Genome Atlas;

LNM

lymph node metastasis(LNM)

OS

overall survival;

OD

optical density.

\section{Declarations}

\section{Ethics approval and consent to participate}

Breast cancer tissues were taken from patients undergoing surgical resection at the Harbin Medical University Cancer Hospital (Harbin, China). All work was approved by the Ethics Committee of Harbin Medical University. All animal work was performed in compliance with the Institutional Animal Care and Use Committee (IACUC) of the second affiliated hospital of Harbin medical university in China and the $\mathrm{NIH}$ Guide for the Care and Use of Laboratory Animals.

\section{Consent for publication}

We have obtained consents to publish this paper from all the participants of this study.

\section{Availability of data and materials}

All data generated or analysed during this study are included in this published article [and its supplementary information files].

\section{Competing interests}

The authors declare that they have no competing interests.

\section{Acknowledgements}

Not applicable.

\section{Funding}

This work was supported by National Natural Science Foundation of China (No.81773295 and 81573001) to Xuesong Chen

\section{Authors' contributions}


$\mathrm{CL}, \mathrm{CX}$ and $\mathrm{LY}$ designed this research. LY carried out most of the experiments, analyzed the data, drew the figures and drafted this manuscript. LM, GJ, CY and JX helped with cell culture, western blot experiments and the CCK-8 assay. WD and JY helped with the IHC assay. YQ, GY and LY helped with the animal experiment. $\mathrm{CX}$ helped check the manuscript and figures. All authors read and approved the final manuscript.

\section{References}

1. Siegel RL, Miller KD, Jemal A. Cancer Statistics. 2017. CA Cancer J Clin. 2017;67:7-30.

2. Chen W, Zheng R, Baade PD, Zhang S, Zeng H, Bray F, et al. Cancer statistics in China, 2015. CA Cancer J Clin. 2016;66:115-32.

3. Benns MV, Luk T, Scoggins CR. Surgical prophylaxis for inheritable malignant diseases: breast cancer and endocrine disease. Am Surg. 2009;75:529-36.

4. Guarneri V, Conte P. Metastatic breast cancer: therapeutic options according to molecular subtypes and prior adjuvant therapy. Oncologist. 2009;14:645-56.

5. Massague J, Obenauf AC. Metastatic colonization by circulating tumour cells. Nature. 2016;529:298-306.

6. Holohan C, Van Schaeybroeck S, Longley DB, Johnston PG. Cancer drug resistance: an evolving paradigm. Nat Rev Cancer. 2013;13:714-26.

7. Mizushima N. The pleiotropic role of autophagy: from protein metabolism to bactericide. Cell Death Differ.2005 12Suppl 2:1535-41.

8. Zhu JH, Horbinski C, Guo F, Watkins S, Uchiyama Y, Chu CT. Regulation of autophagy by extracellular signal-regulated protein kinases during 1-methyl-4-phenylpyridiniuminduced cell death. Am J Pathol. 2007;170(1):75-86.

9. Li X, Li Y, Fang S, Su J, Jiang J, Liang B, et al. Downregulation of autophagy-related gene ATG5 and GABARAP expression by IFN-lambda1 contributes to its anti-HCV activity in human hepatoma cells. Antiviral Res. 2017;140:83-94.

10. Garcia-Mariscal A, Li H, Pedersen E, Peyrollier K, Ryan KM, Stanley A, et al. Loss of RhoA promotes skin tumor formation and invasion by upregulation of RhoB. Oncogene. 2018;37:847-60.

11. Kabeya Y, Mizushima N, Yamamoto A, Oshitani-Okamoto S, Ohsumi Y, Yoshimori T. LC3, GABARAP and GATE16 localize to autophagosomal membrane depending on form-ll formation. J Cell Sci. 2004;117:2805-12.

12. Cook JL, Re RN, deHaro DL, Abadie JM, Peters M, Alam J. The trafficking protein GABARAP binds to and enhances plasma membrane expression and function of the angiotensin II type 1 receptor. Circ Res. 2008;102:1539-47.

13. Lainez S, Valente P, Ontoria-Oviedo I, Estevez-Herrera J, Camprubi-Robles M, Ferrer-Montiel A, et al. GABAA receptor associated protein (GABARAP) modulates TRPV1 expression and channel function and desensitization. FASEB J. 2010;24:1958-70. 
14. Rho SB, Byun HJ, Kim BR, Kim IS, Lee JH, Yoo R, et al. GABAA receptor-binding protein promotes sensitivity to apoptosis induced by chemotherapeutic agents. Int J Oncol. 2013;42:1807-14.

15. Hu H, Asweto CO, Wu J, Shi Y, Feng L, Yang X, et al. Gene expression profiles and bioinformatics analysis of human umbilical vein endothelial cells exposed to PM2.5. Chemosphere. 2017;183:58998.

16. Park SH, Kim BR, Lee JH, Park ST, Lee SH, Dong SM, et al. GABARBP down-regulates HIF-1alpha expression through the VEGFR-2 and PI3K/mTOR/4E-BP1 pathways. Cell Signal. 2014;26:1506-13.

17. Haddadi N, Lin Y, Travis G, Simpson AM, Nassif NT. McGowan EM. PTEN/PTENP1: 'Regulating the regulator of RTK-dependent PI3K/Akt signalling', new targets for cancer therapy. Mol Cancer. 2018;17:37.

18. Wang J, Ren D, Sun Y, Xu C, Wang C, Cheng R, et al. Inhibition of PLK4 might enhance the anti-tumour effect of bortezomib on glioblastoma via PTEN/PI3K/AKT/mTOR signalling pathway. J Cell Mol Med. 2020.

19. Sun K, Luo J, Guo J, Yao X, Jing X, Guo F. The PI3K/AKT/mTOR signaling pathway in osteoarthritis: a narrative review. Osteoarthritis Cartilage. 2020;28:400-9.

20. Larue L, Bellacosa A. Epithelial-mesenchymal transition in development and cancer: role of phosphatidylinositol 3' kinase/ AKT pathways. Oncogene. 2005;24:7443-54.

21. Christofori G. New signals from the invasive front. Nature. 2006;441:444-50.

22. Thiery JP. Epithelial-mesenchymal transitions in tumour progression. Nat Rev Cancer. 2002;2:44254.

23. Zeisberg M, Neilson EG. Biomarkers for epithelial-mesenchymal transitions. J Clin Invest. 2009;119:1429-37.

24. Thiery JP. Epithelial-mesenchymal transitions in development and pathologies. Curr Opin Cell Biol. 2003;15:740-6.

25. Chen X, Meng Q, Zhao Y, Liu M, Li D, Yang Y, et al. Angiotensin II type 1 receptor antagonists inhibit cell proliferation and angiogenesis in breast cancer. Cancer Lett. 2013;328:318-24.

26. Lin Q, Chen X, Meng F, Ogawa K, Li M, Song R, et al. ASPH-notch Axis guided Exosomal delivery of Prometastatic Secretome renders breast Cancer multi-organ metastasis. Mol Cancer. 2019;18:156.

27. Wang Y, Zhao L, Han X, Wang Y, Mi J, Wang C, et al. Saikosaponin A Inhibits Triple-Negative Breast Cancer Growth and Metastasis Through Downregulation of CXCR4. Front Oncol. 2019;9:1487.

28. Sasai M, Sakaguchi N, Ma JS, Nakamura S, Kawabata T, Bando H, et al. Essential role for GABARAP autophagy proteins in interferon-inducible GTPase-mediated host defense. Nat Immunol. 2017;18:899-910.

29. Gugnoni M, Sancisi V, Gandolfi G, Manzotti G, Ragazzi M, Giordano D, et al. Cadherin- 6 promotes EMT and cancer metastasis by restraining autophagy. Oncogene. 2017;36(5):667-77.

30. Akalay I, Janji B, Hasmim M, Noman MZ, André F, De Cremoux P, et al. Epithelial-to-mesenchymal transition and autophagy induction in breast carcinoma promote escape from T-cell-mediated lysis. 
Cancer Res.2013 73(8):2418-27.

31. Wu Z, Lu H, Yao J, Zhang X, Huang Y, Ma S, et al. GABARAP promotes bone marrow mesenchymal stem cells-based the osteoarthritis cartilage regeneration through the inhibition of PI3K/AKT/mTOR signaling pathway. J Cell Physiol. 2019;234:21014-26.

\section{Figures}

a

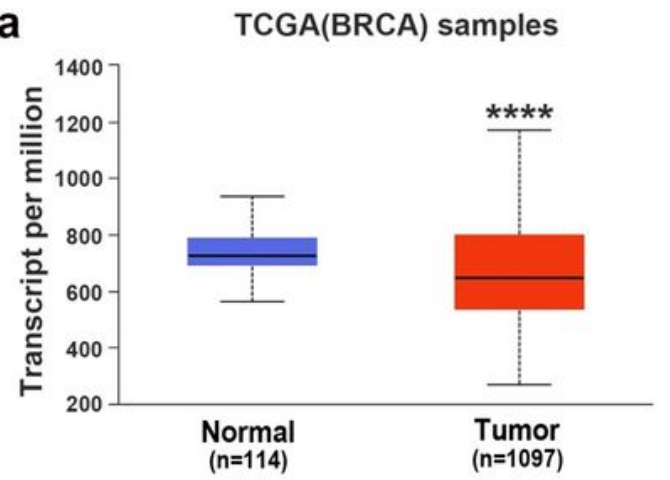

C

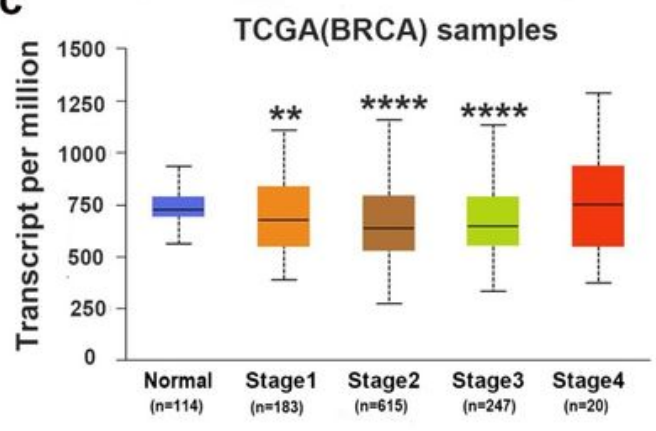

b

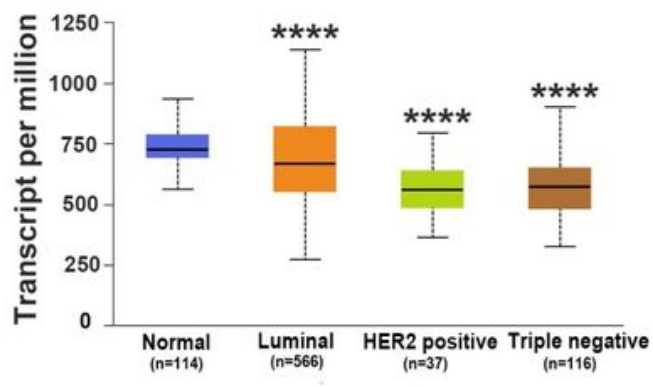

d

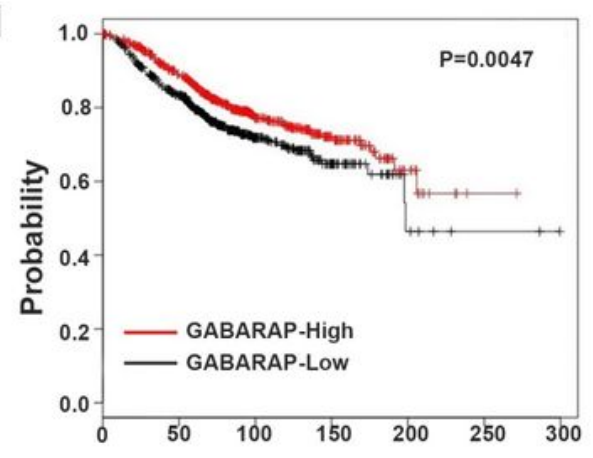

e

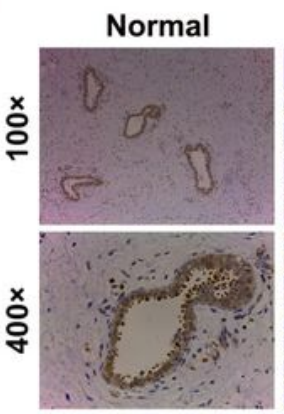

DCIS

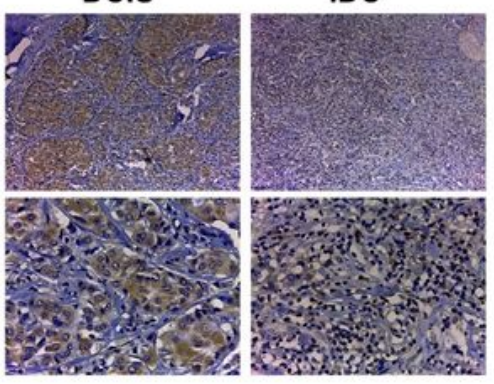

f

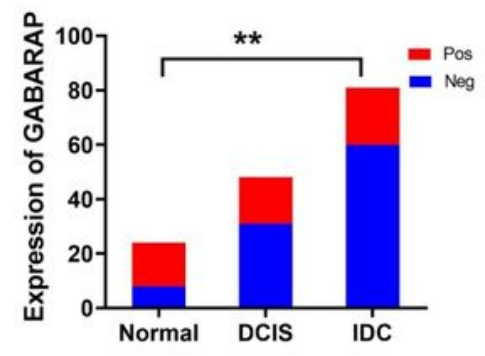

g

h

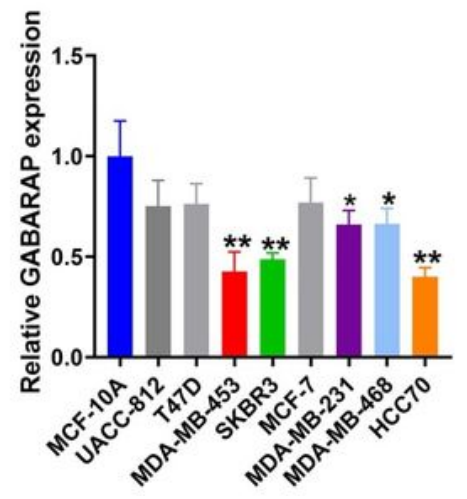




\section{Figure 1}

GABARAP is downregulated in breast cancer specimens and cell lines. a. Expression profile of GABARAP in primary breast cancer tissues $(n=1097)$ and normal breast tissues $(n=114)(T C G A)$. b. Expression of GABARAP in BRCA based on breast cancer subclasses(TCGA). c. Expression of GABARAP in BRCA based on individual cancer stages(TCGA). d. Overall survival of breast cancer patients according to GABARAP expression(TCGA). e. Representative images of GABARAP immunohistochemical staining. $f$. A statistically significant of 'positive' or 'negative' GABARAP staining in 87cases of IDC, 48 cases of DCIS and 24 cases of normal breast tissue. g. Western blotting analysis of GABARAP expression in 8 human breast cancer cell lines and non-transformed MCF-10A. h. A statistically significant of GABARAP expression in 8 human breast cancer cell lines and non-transformed MCF-10A. $\left({ }^{*} P<0.05, * * P<0.01\right.$, $* \star * P$ $<0.001 \star \star \star \star P<0.0001$. TCGA $=$ The Cancer Genome Atlas). 
a

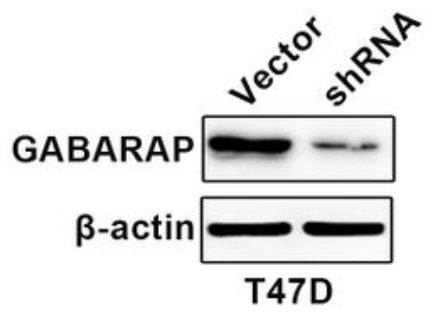

b

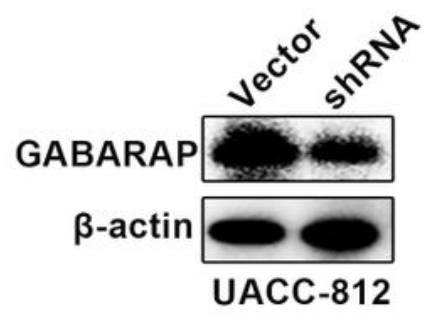

C

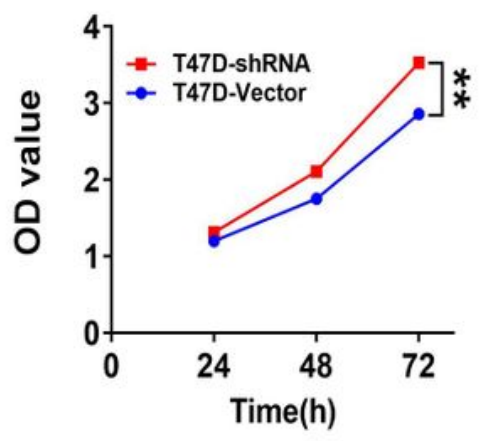

d

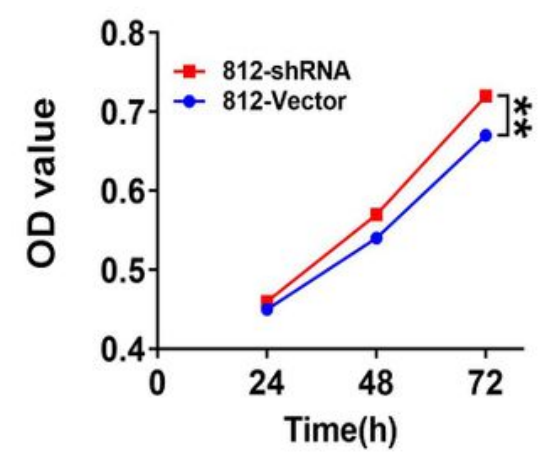

e

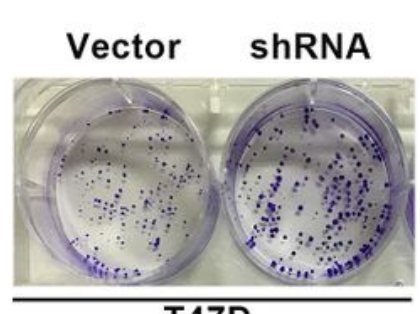

T47D

g

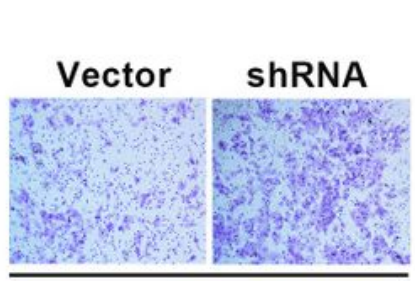

i

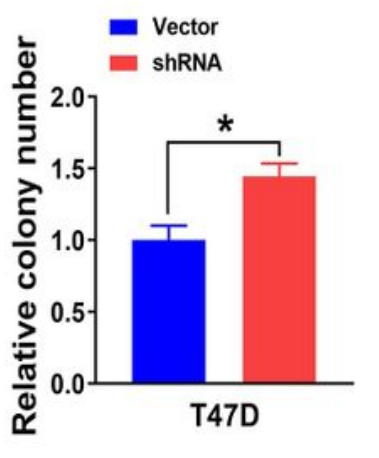

f
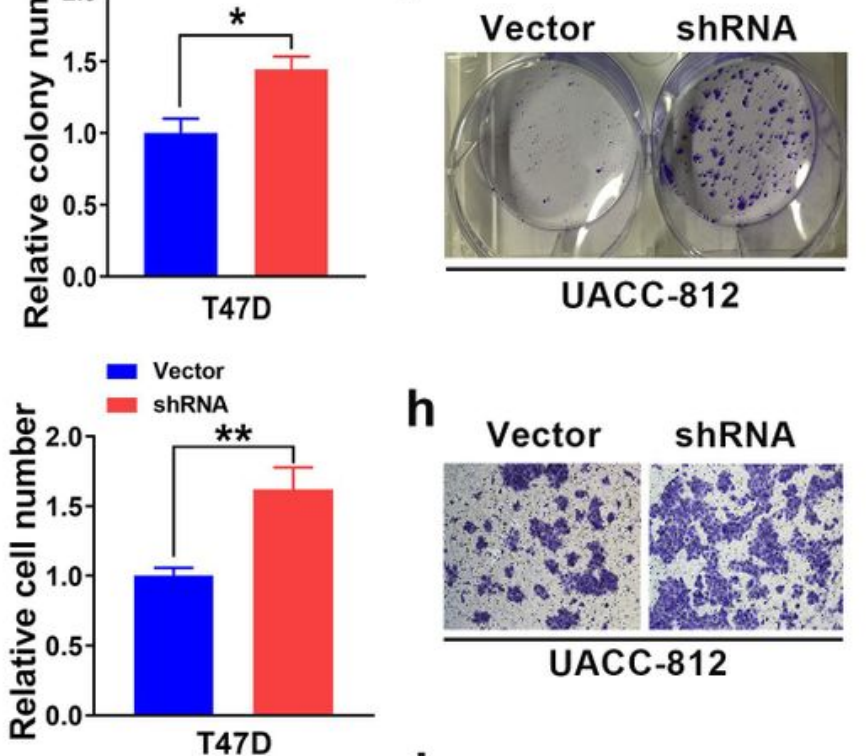

h

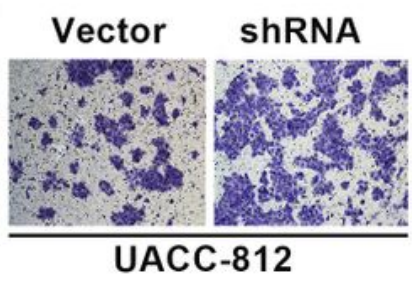

j
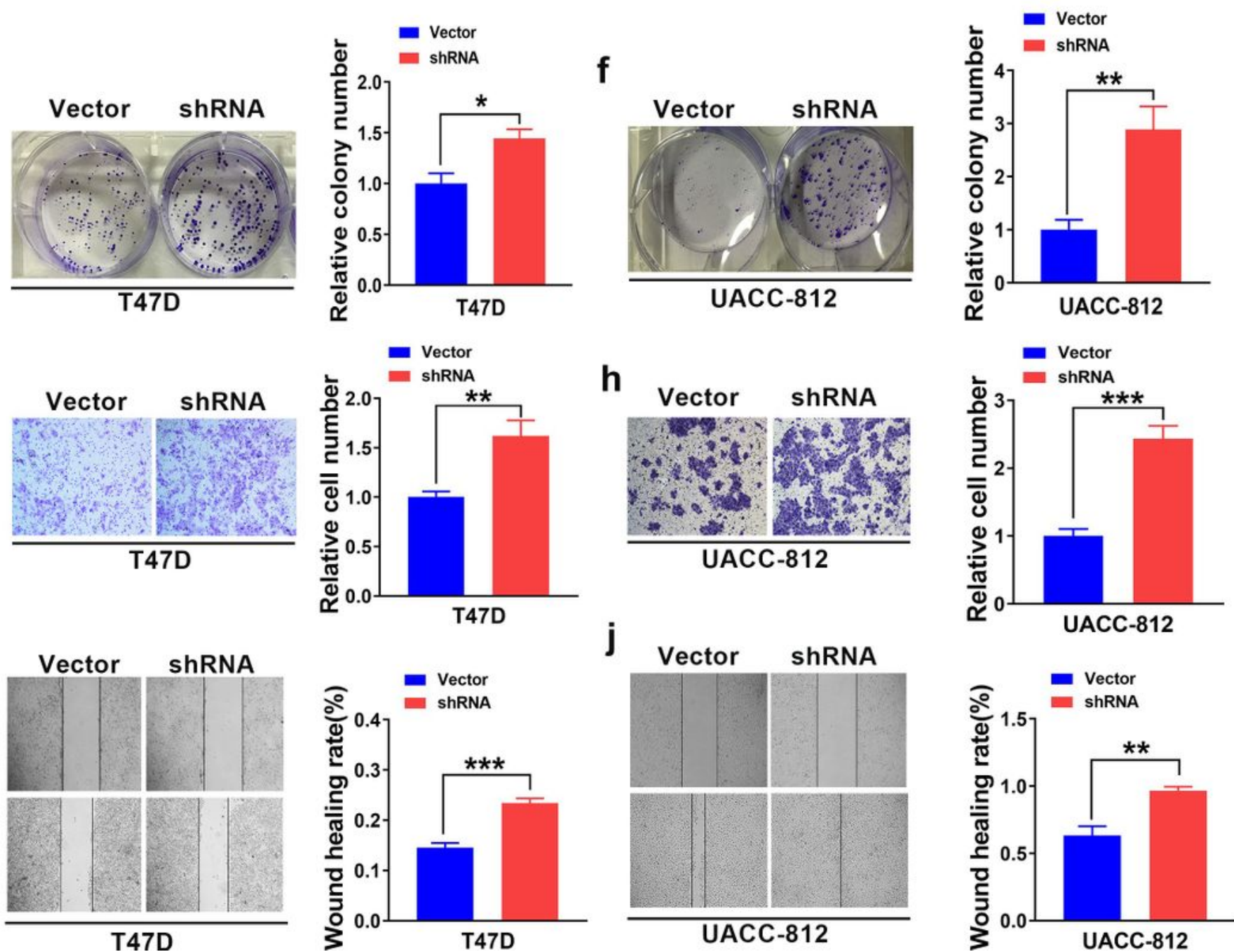

T47D
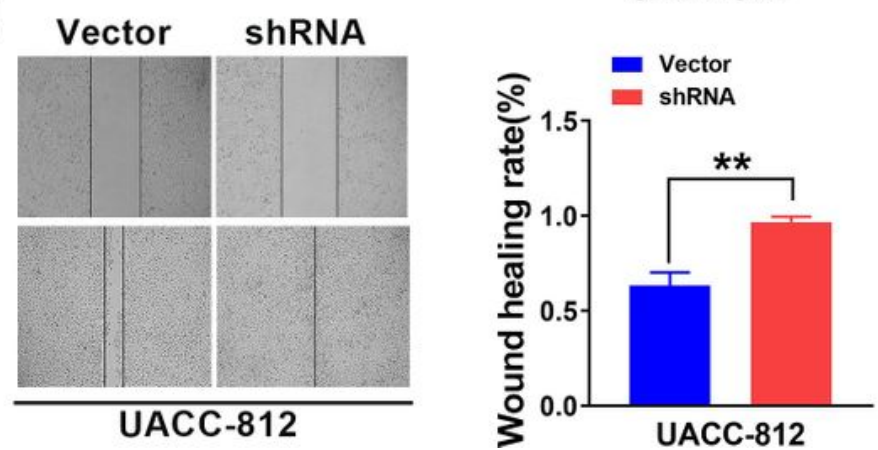

Figure 2

Low GABARAP level enhances the malignant behavior of breast cancer cells. a. Knockdown of GABARAP in T47D, GABARAP expression was determined using Western blot. b. Knockdown of GABARAP in UACC812 cells, GABARAP expression was determined using Western blot. c. Cell proliferation in T47D-vector, T47D-shRNA cells was detected by CCK-8 assays. d. Cell proliferation in 812-vector, 812- shRNA cells was detected by CCK-8 assays. e. Colony-forming efficiency was determined in T47D-vector, T47D-shRNA 
cells. f. Colony-forming efficiency was determined in 812-vector, 812- shRNA cells. g. Invasive abilities conveyed by T47D-vector, T47D-shRNA cells were measured by Matrigel invasion assays. h. Invasive abilities conveyed by 812 -vector, 812 - shRNA cells were measured by Matrigel invasion assays. i.

Migration ability conveyed by T47D-vector, T47D-shRNA cells were assessed by wound-healing migration assays. j. Migration ability conveyed by 812-vector, 812- shRNA cells were assessed by wound-healing migration assays. ( $* \mathrm{P}<0.05, * * \mathrm{P}<0.01$, $* * * \mathrm{P}<0.001$ )

a

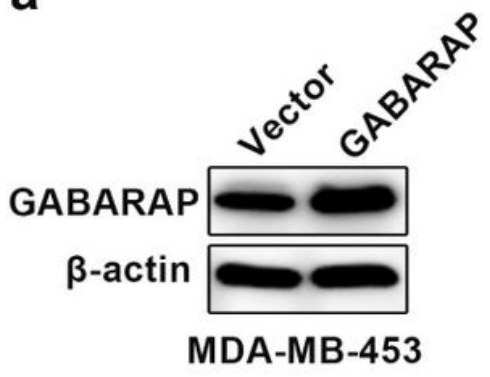

C

\section{Vector GABARAP}

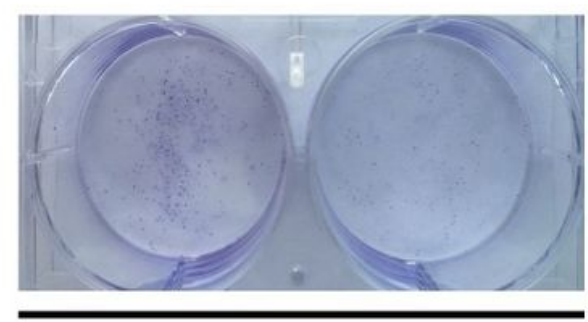

MDA-MB-453

d

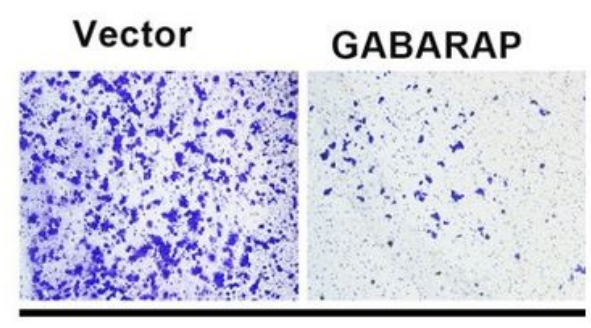

MDA-MB-453 b
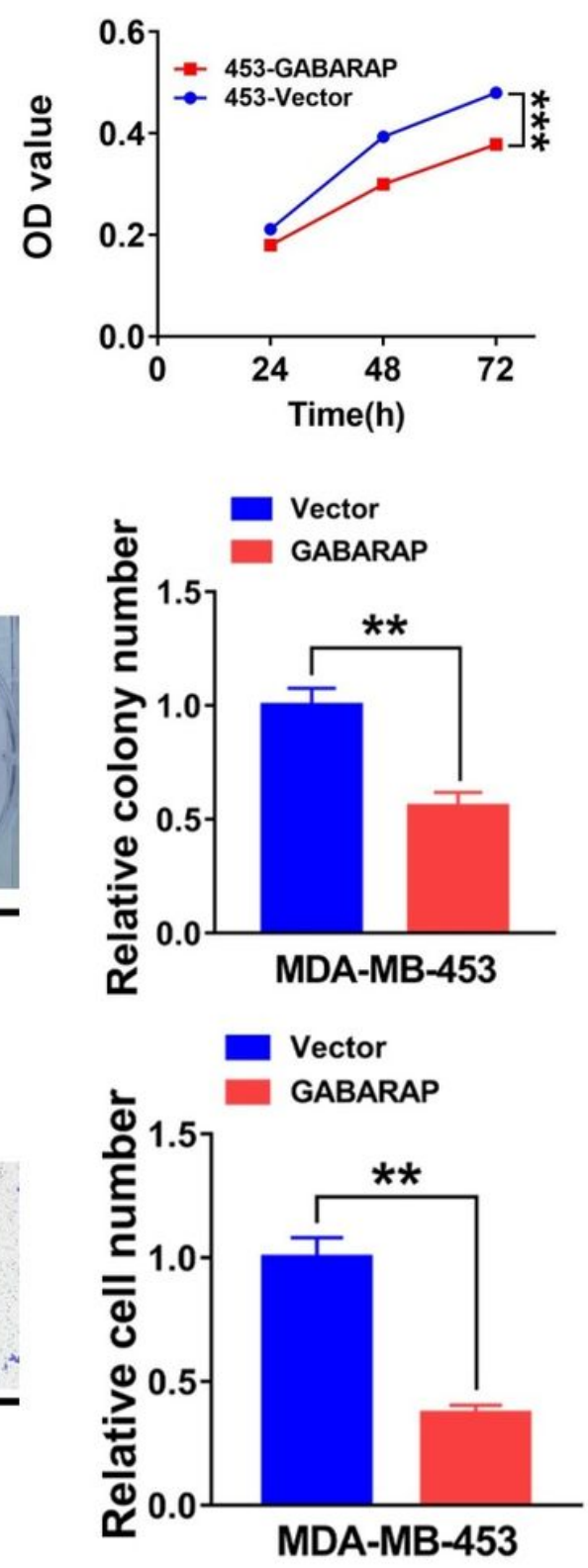

e
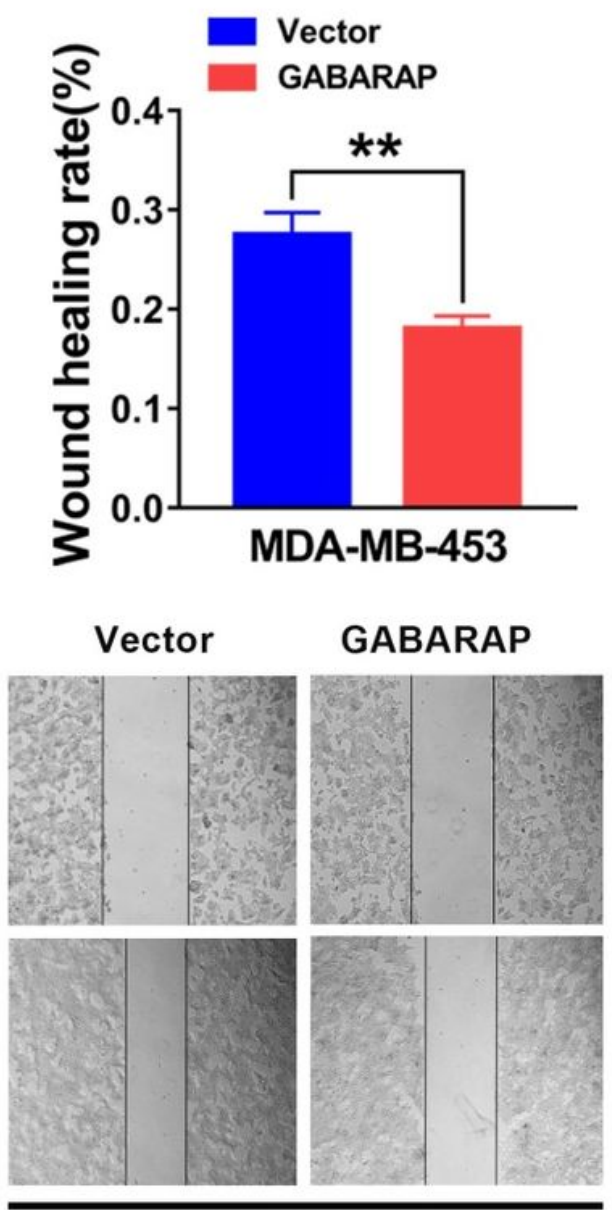

MDA-MB-453

Figure 3

High GABARAP level suppresses the malignant behavior of breast cancer cells a. Over-expression of GABARAP in MDA-MB-453 cells, GABARAP expression was determined using Western blot. b. Cell proliferation in 453-vector, 453-GABARAP cells was detected by CCK-8 assays. c. Colony-forming efficiency was determined in 453-vector, 453-GABARAP cells. d. Invasive abilities conveyed by 453-vector, 
453-GABARAP cells were measured by Matrigel invasion assays. e. Migration ability conveyed by 453vector, 453-GABARAP cells were assessed by wound-healing migration assays. ( ${ }^{*} P<0.05,{ }^{*} P<0.01$, $\star \star * P<0.001)$

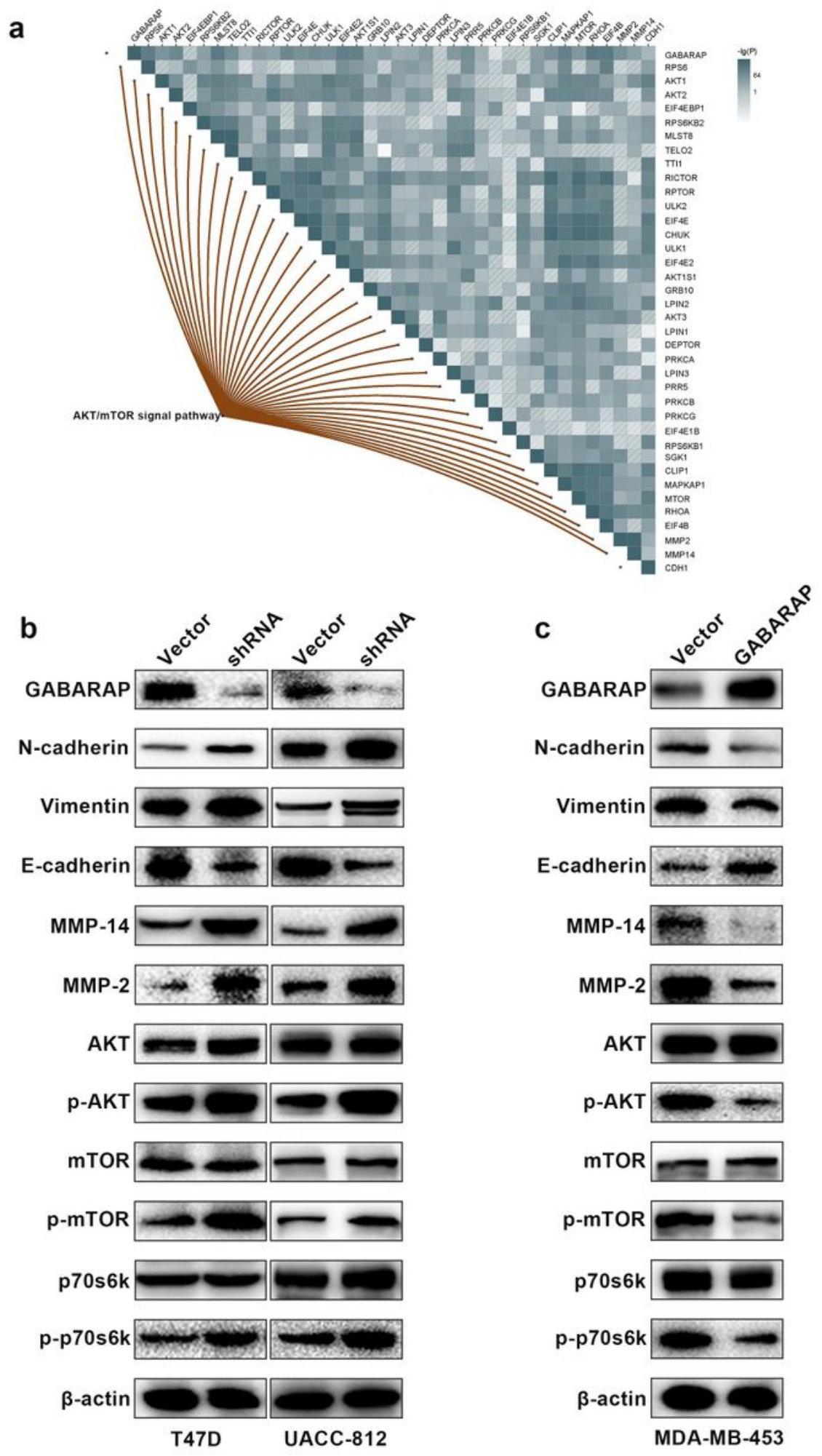

Figure 4

Low GABARAP level promotes cellular EMT via AKT/mTOR signaling in breast cancer. a. Pearson correlation was calculated among genes related to GABARAP, CDH1, MMP2, MMP14 and Akt/ mTOR 
signaling pathway in breast cancer patients clinical cohort (TCGA). b. Western blot analyses were used to detect the expression levels of E-cadherin, N-cadherin, Vimentin, MMP2, MMP14 p-AKT, AKT, P-mTOR, mTOR, p-p70s6k and p70s6k in T47D-vector, T47D-shRNA, 812-vector, 812- shRNA cells. c. Western blot analyses were used to detect the expression levels of E-cadherin, $\mathrm{N}$ - cadherin, Vimentin, MMP2, MMP14 pAKT, AKT, P-mTOR, mTOR, p-p70s6k and p70s6k in 453-vector, 453-GABARAP cells.

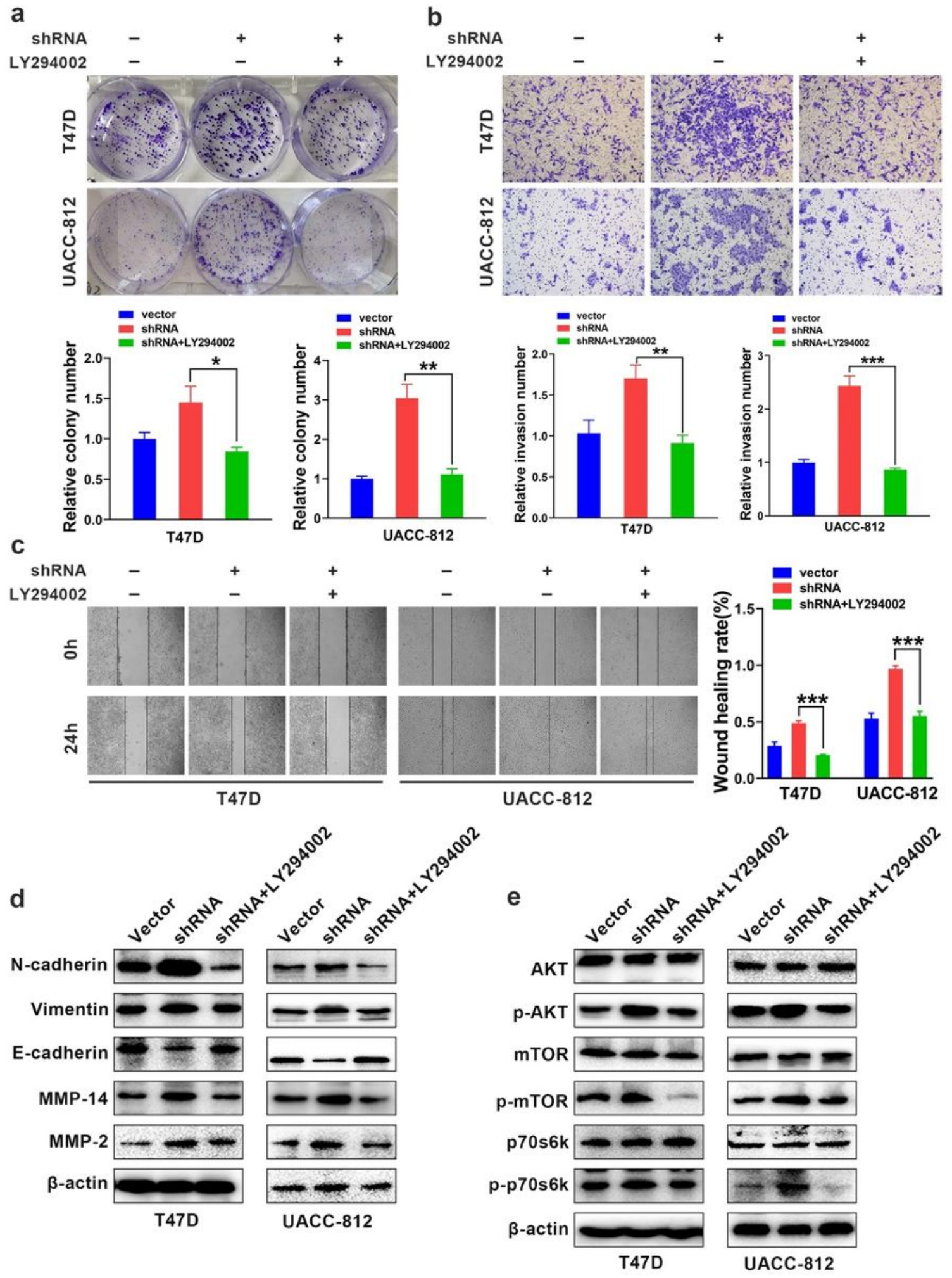

Figure 5 
LY-294002(AKT pathway inhibitor) reverses GABARAP-inhibited proliferation, invasion, migration and EMT. a. Colony-forming efficiency were performed in T47D-Vector, T47D-shRNA, T47D-shRNA cells incubated with LY-294002, 812-vector, 812- shRNA, 812- shRNA cells incubated with LY-294002. b. Invasion assays were performed in the indicated cells. c. Migration assays were performed in the indicated cells. $d$. Western blot analyses were used to detect the expression levels of E-cadherin, $\mathrm{N}$ cadherin, Vimentin, MMP2 and MMP14 in the indicated cells. e. Western blot analyses were used to detect the expression levels of p-AKT, AKT, P-mTOR, mTOR, p-pl3k and pl3k in the indicated cells.

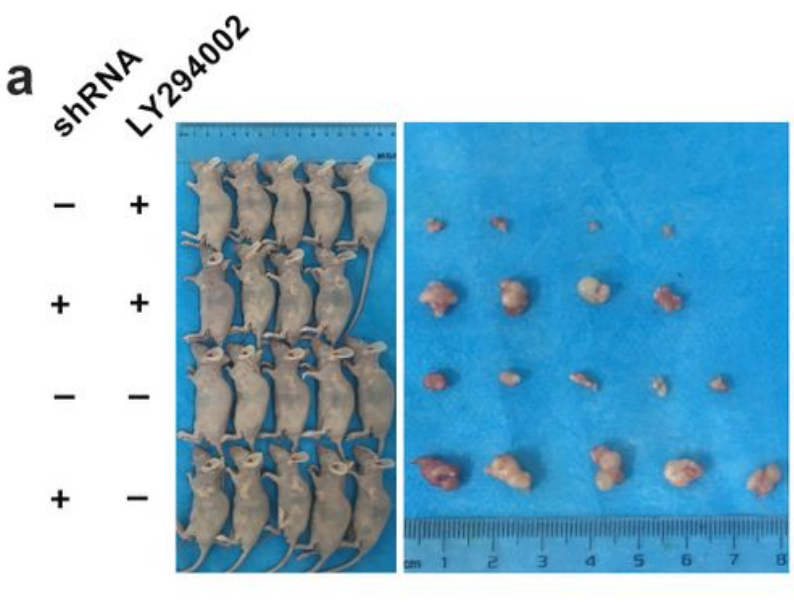

b

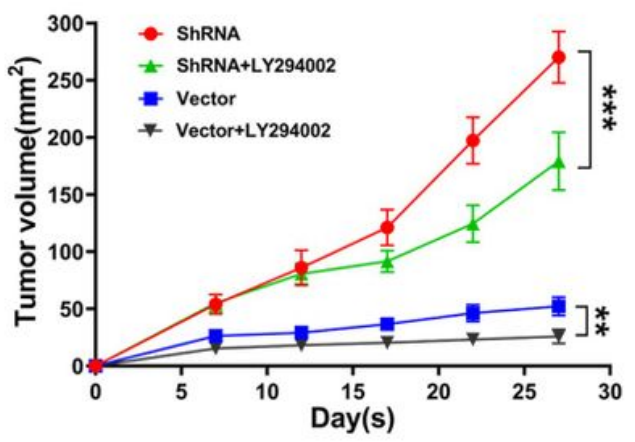

C
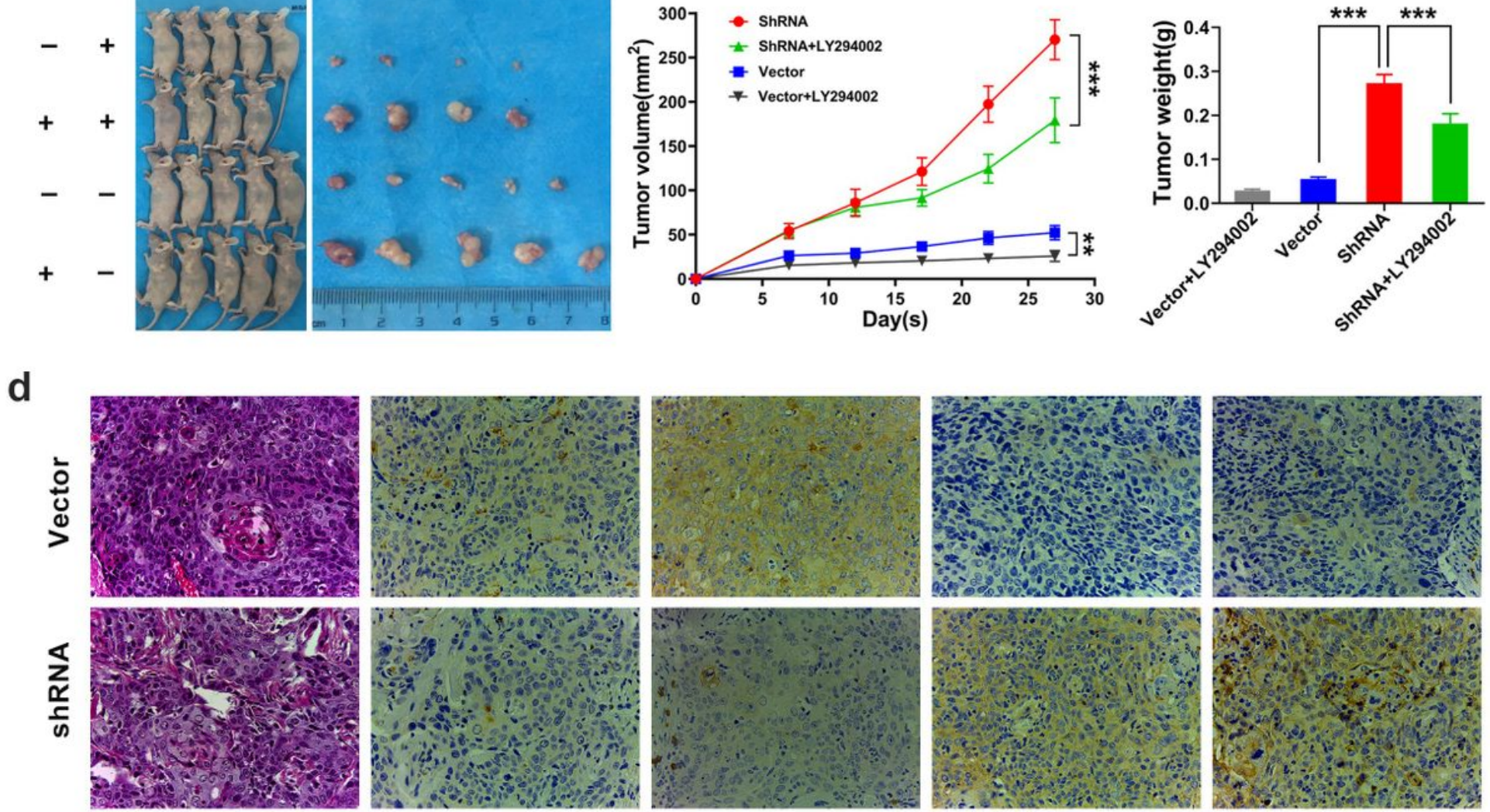

HE
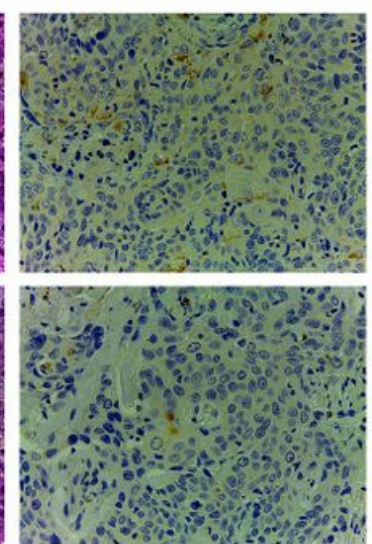

GABARAP

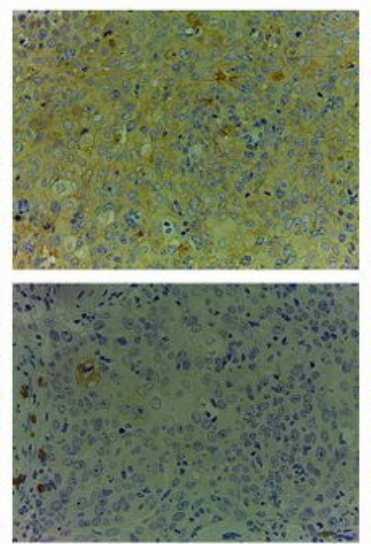

E-cadherin
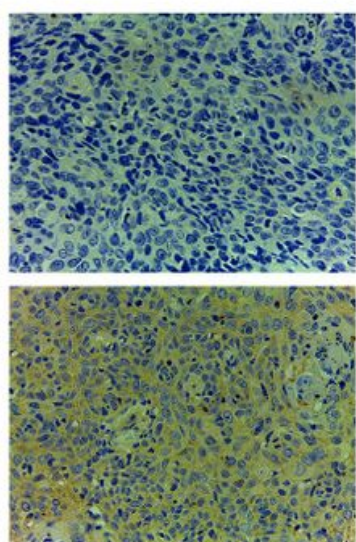

MMP2
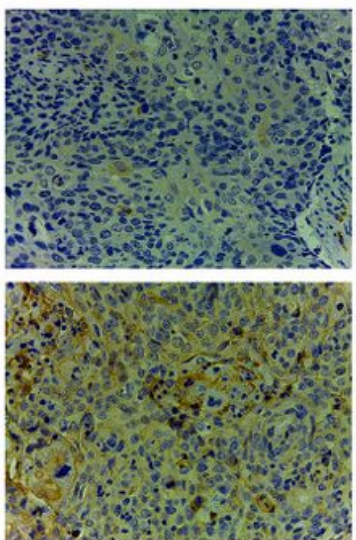

p-mTOR

\section{Figure 6}

GABARAP suppresses breast cancer progression in vivo. a. Representative images of nude mice and tumors at day 28 after inoculation of UACC-812 cells with or without shRNA mediated silencing of GABARAP. Vector control group, mice inoculated with control UACC-812 cells; GABARAP -shRNA group, mice inoculated with GABARAP silenced UACC-812 cells; Vector control + LY294002 group, mice inoculated with control UACC-812 cells and treated with LY294002; GABARAP -shRNA + LY294002 group, mice inoculated with GABARAP silenced UACC-812 cells and treated with LY294002. b. Tumor growth curves in 4 groups of nude mice. $\mathrm{N}=5$ for 1 ), 2) and 3) groups, $\mathrm{N}=4$ for 4 )group. $\mathrm{c}$. The tumor weights were measured, and the data are presented as the means \pm SDs. d. Immunostaining of proteins in tumors 
from the vector control group and GABARAP-shRNA group. First column, H\&E staining; second column, immunostaining for GABARAP ; third column, immunostaining for E-cadherin; fourth column, immunostaining for MMP2; fifth column, immunostaining for $p$-mTOR. Magnification, $400 \times .\left({ }^{\star} p<0.05\right.$; $\left.{ }^{* *} \mathrm{p}<0.01 ; * \star * \mathrm{p}<0.001\right)$.

a

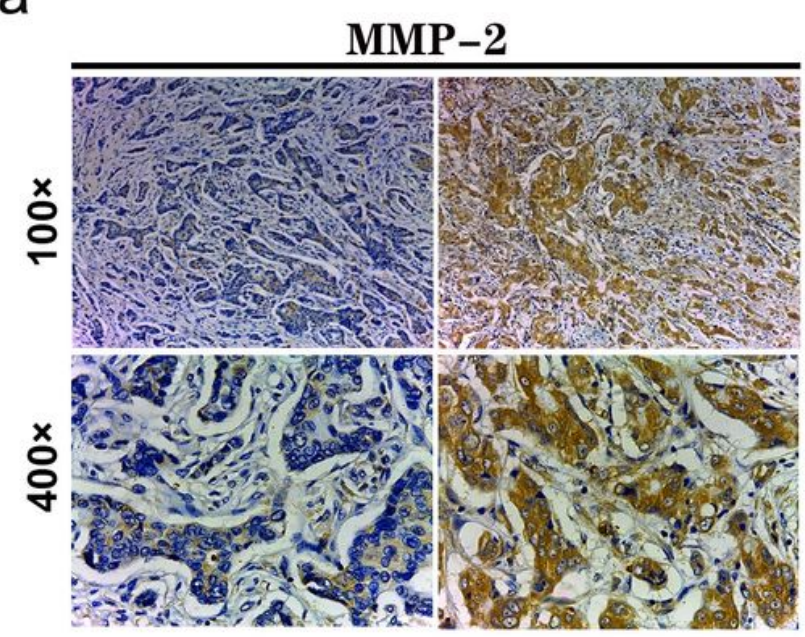

C

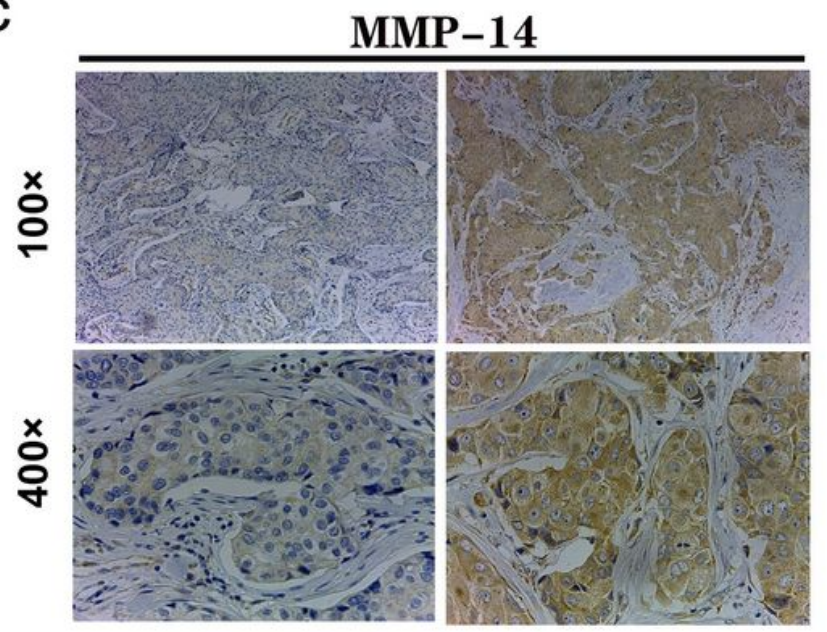

b
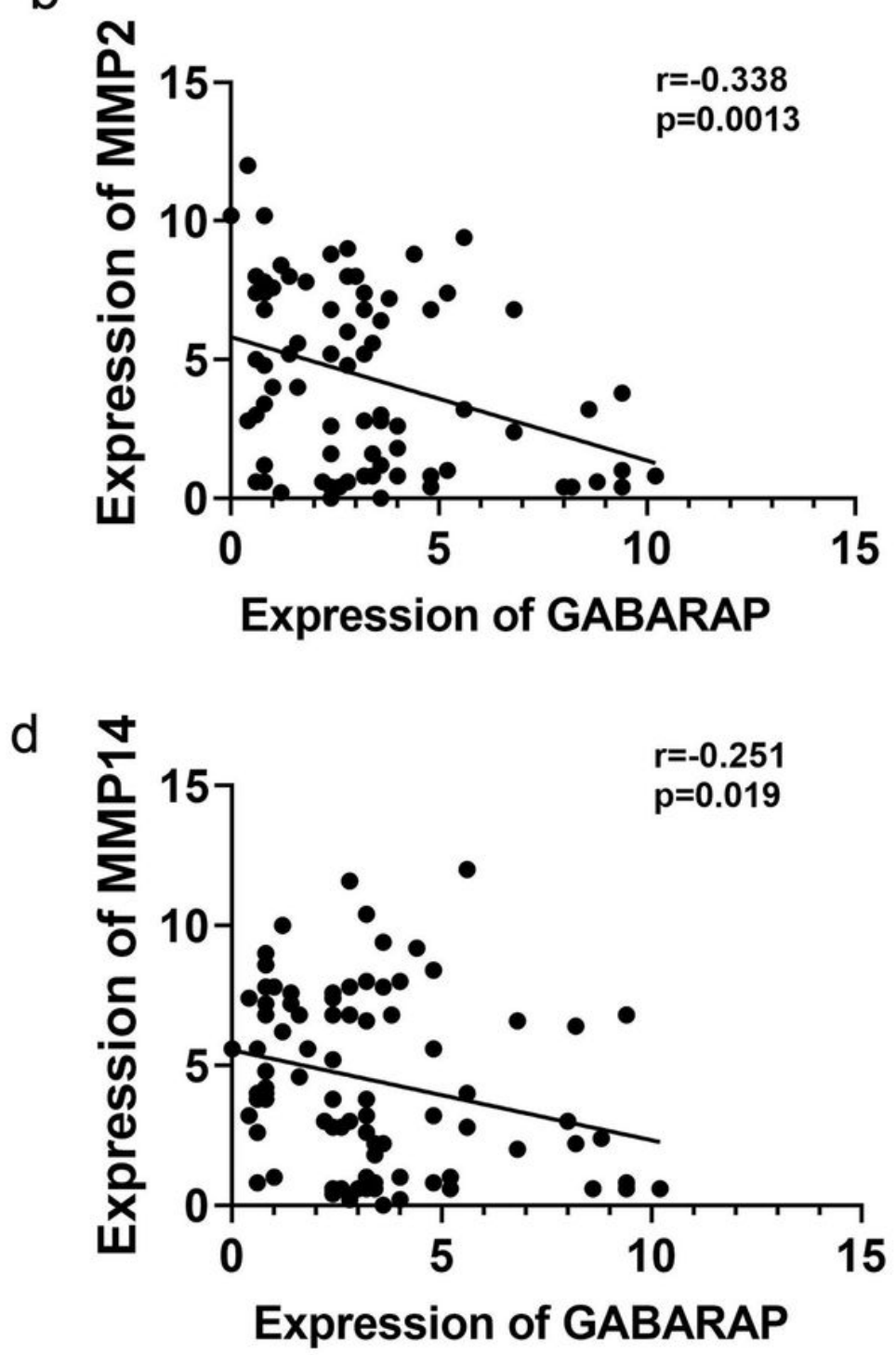

Figure 7

Correlation between MMPs and GABARAP expression in breast cancer tissues from patients. a. Representative immunostaining profiles of MMP2 in GABARAP low expression and GABARAP high expression breast cancer tissues. Magnification, 400x. b. Correlation analysis of the expression of MMP2 and GABARAP using the pearson correlation coefficient. GABARAP negatively correlated with MMP2 at the protein level. c. Representative immunostaining profiles of MMP14 in GABARAP low expression and GABARAP high expression breast cancer tissues. Magnification, 400x. d. Correlation analysis of the expression of MMP14 and GABARAP using the pearson correlation coefficient. GABARAP negatively correlated with MMP14 at the protein level. 


\section{Supplementary Files}

This is a list of supplementary files associated with this preprint. Click to download.

- SupplementaryMaterial.docx 